\title{
ZUR LEXICOLOGIE DES ALTFRIESISCHEN.
}

Der altfriesische wortschatz ist nur zu einem kleinen teile auf uns gekommen; ja wir kennen eigentlich nur die rechtssprache des stammes genauer, da die erhaltene altfriesische litteratur fast ausschliesslich aus rechtsaufzeichnungen besteht. Vermebren lässt sich der in den rechtsquellen enthaltene wortschatz durch die orts- und flurnamen, deren in alten guterregistern und schenkungsurkunden sehr viele genannt werden. Freilich sind die meisten derselben noch nicht gentigend erklärt. Aber auch sonst stösst man hier und da auf ein altfriesisches wort, das in den altfriesischen wörterbuchern noch fehlt. Einige solcher wörter stehen in dem VII. capitel der Traditiones Fuldenses .(in der ausgabe von Dronke (1844) s. 45, im 0stfriesischen urkundenbuche II s. 787) und zwar im eingange jenes abschnittes, der uberschrieben ist: 'Iste sunt solutiones uirorum in Fresia qui censum solvere debent'. Diese stelle ist wie das ganze VII. capitel, welches die einkunfte bebandelt, die das kloster aus Friesland bezog, durch schreibfehler arg entstellt, die sich aber als solche von dem mit der geschichte des landes vertrauten ziemlich leicht erkennen lassen. Ich stelle hier neben den handschriftlichen text der stelle einen von den fehlern gesäuberten text, in welchem ich die friesisischen worte hervorhebe, und bespreche dann diese worte sowie die textverbesserungen im einzelnen.

Handschriftlicher text:

(§31) $\left.{ }^{1}\right)$ De possessione Gebes censum quem Nordalah comes et aduocatus Fresonum constituit. hoc
Verbesserter text:

(§31) De possessione Gebes censum quem Nordalah, comes et aduocatus Fresonum, constituit, hoc

1) Wegen des citierens empfiehlt es sich, die von Dronke herrührende einteilung der capitel des registers in paragraphen beizubehalten. 
est in Lanthusa uilla .XII. den. et XX solidi. secundus census ad siceram emendam .X. den. Tercius census ad herbam soluendam .XXX. den. Quod est apud Fresones rosbannare, id est, ut equi commune pabulum habeant in prato post abscisionem feni. ab omnibus datur constitutus census. Quartus census qui dicitur rûtforst .X. denarii. Naschfelden de duobus unus census constitutus est. qui census erit XXX. et bis. X.

(§ 32) De possessione Gerwihi lantstûre soluendam .X. den. Ad siceram emendam .X. den. Heribannum soluendum .I. ad rosbannum XXX den. rutforstar. $X$ den. Hic census adhuc est.

(§ 33) De ministerio Luterichi. VII pondera frumenti. et duas uncias et $X$. den.

(§ 34) De ministerio einungi .VIIII. pondera frumenti. et XXX denarii. exceptis nascpendinge.

1. Lanthura. Ein dorf 'Lanthusa' gab es im westlauwerschen Friesland, uber welches Nordalah, ein nachkomme des friesenkönigs Ratbod, seit dem jabre 793 als graf waltete, nicht. Der name wăre auch für ein dorf geradezu unmöglich. Dass nun gar ein zins danach benannt worden wäre, ist unsinnig. Was gemeint ist, zeigt der ausdruck 'lantstûre soluendam' in § 32. Aus lanthura bat der schreiber Lanthusa gemacht. Er verwechselt $s$ und $r$ sehr häufig; so schreibt er z. b. 'uidemus' statt 'uidemur' (Dronke a. a. o. $\$ 108)$, 'Wisaha' statt 'Wiraha' (§ 13), 'More' fur 'Mose' (§ 17), 'Wacheringe' für 'Wachesinge' (§ 60), 'Emergewe' für 'Emesgewe' (§ 62), 'Hunergewe' fur 'Hunesgewe' (§ 89), 'Heterheim' für 'Hetesheim' (§ 103). Da er sein 'Lanthusa' für einen ort ansah, musste er naturlich noch ein in davorsetzen. Auch lantstare in $\$ 32$ muss in lanthure gebessert werden. Die Friesen bezeichnen den pachtzins niemæls als 'steuer', sondern stets als hûre oder hêre. Das westlauwersche landhure bedeutet den grundzins, die feldpacht. Das im 11. jahrb. abgefasste, aber in einem texte des 15. jahr- 
hunderts uberlieferte westlauwersche schulzenrecht braucht dafür landhêre (v. Richth., Fries. Rq. 395, 18), welches zum emsgauischen londhêre $(195,13 ; 209,19)$ stimmt. Dass aber auch die form hure altfriesisch ist, geht aus einer urkunde des abtes Hadamar von Fulda vom jahre 945 hervor: 'omnia que ipsi (sc. Fresones) nominant forahura' (Dronke s. 68, Ostfries. urkb. II 8. 793). Auch forahûre fehlt im v. Richthofenschen wörterbuche. Das wort bedeutet dasselbe wie das ostfriesische formîde, nämlich eine bei der verpachtung der grundstucke $\mathrm{zu}$ zahlende vorausgabe. Ueber diese formîde, ein wort, das ebenfalls bei v. Richthofen fehlt, belehrt folgende interessante stelle im zweităltesten Werdener guterregister (10. oder 11. jahrh.), welche von verpachteten gutern handelt, die das kloster im ostlauwerschen Friesland besass: 'omni anno in festo sancti Jacobi ad iustam huram dabuntur LXII talenta, quorum dimidietas in palliis datur, altera pars in denariis. In tercio autem anno ... XXXVI arietes. Post quintum uero annum in sexto terrae tocius erit redemptio sicut inueniri potest in placito nuntii abbatis et monasterii, quod uocatur formida, nisi forte infra istos annos aliquis moriatur, pro cuius terra alius aliquis formidam statim det, antequam tempus ueniat formidae, quam omnes dabunt predicto sexto anno' (Crecel. Ind. Werd. s. 19, Ostfries. urkb. II s. 773). Diese formîde oder, wie sie bei den westlauwerschen Friesen hiess, forahture wurde also nur beim abschluss bez. bei der erneuerung des pachtvertrages gezahlt, wahrend landhare oder landhêre die alljahrlich zu entrichteude feldpacht war.

2. Nasc-scelde, nasc-pendinge. Der zweite zins wird als 'census ad siceram emendam' bezeichnet. Er ist also eine zum kauf des lith, das bekanntlich beim feierlichen abschluss von kauf- und pachtvertrăgen getrunken wurde, also eine zum lithkap oder wînkap (vgl. v. Richth., Altfries. wörterb. 1151) zu erlegende abgabe. Sie fălt mit jener forahure bez. formîde zusammen. Sie betrug nun, wie sich aus $\$ \S 31.32$ ergibt, 10 pfennige, der dritte zins dagegen 30 , der vierte wider 10 pfennige. Nach dem schlusssatze von $\$ 31$ hatte man diese drei abgaben, die von jedem pachtgute in gleicher höhe gezahlt wurden, zu einer abgabe von 50 pfennigen verbunden. In diesem satze erfahren wir den friesischen namen des 
zweiten zinses. Das handschriftliche 'naschfelden' ist freilich sinnlos, das $n$ am schluss des wortes ist aus et verlesen, das dem sinne nach vor de duobus gestanden haben muss. Wie die erste silbe des wortes lauten muss, ersehen wir aus § 34, wo dieselbe abgabe als nasc-pendinge 'nasch-pfennige' bezeichnet wird. Das $h$ in dem verderbten naschfelden ist aus $s c$ verlesen, so wie ja in $\S 32$ umgekehrt st, das in den handschriften jener zeit dem $s c$ zum verwechseln ähnlich sieht, aus $h$ verlesen ist (lantstûre aus lanthure). Da der schreiber mit dem naschelde nichts anzufangen wusste, schob er das $f$ hinter $h$ ein und erhielt so ein naschfeld, das hier gar keinen sinn gibt. Der zins biess nasc-scelde 'nasch-schuld', wofur auch dio bezeichnung nasc-pendinge 'nasch-pfennige' gebraucht wurde. Gerade skelde 'schuld' verwenden die Friesen mit vorliebe fur jede art von zins und abgabe, wie man aus den stellen der friesischen rechtsquellen, die v. Richthofen unter klepskelde, kiningskelde, hofskelde, himulskelde gesammelt hat, leicht erseben kann. Die worte nask-skelde und nask-pendinge kommen in den friesischen rechtsquellen nicht vor. $\mathrm{Da}$ sie dasselbe bezeichnen, wie forahare und formîde, nämlich den census ad siceram emendam, den zum lithkâp oder nînkâp zu erlegenden zins, so ist es wichtig sich daran zu erinnern, dass man noch heutzutage in mehreren gegenden Deutschlands statt wein- oder leitkauf auch nasskauf sagt. Das hat mit 'nass' sicher nichts zu tun, sondern ist durch volksetymologie aus nask-kauf entstanden und enthălt als ersten compositionsteil dasselbe wort wie altfr. nask-skelde und nask-pendinge. Da nun aber 'nasskauf', wofur man also richtiger 'naschkauf' sagen wurde, dasselbe wie 'leitkauf' oder 'weinkauf' bedeutet, so muss nask, wie lîth und wîn, das getränk bezeichnen, welches beim abschluss des vertrages getrunken wurde. Von diesem substantivum nask sind mhd. genasche, genesche und geneschelin, welch letzteres ja Berthold von Regensburg geradezu in der bedeutung 'trinkgeld' verwendet, gebildet. Nask-pendinge und nask-skelde bedeuten nichts anderes als 'trinkgeld'; sie bezeichnen die summe, die zur bezahlung des gelages, das beim abschluss des pachtvertrages stattfand, zu erlegen war.

3. Rosban, rosbannare. Der dritte zins wird von allen pächtern gezahlt, damit sie ihre pferde nach dem schnitt 
des grases auf die wiesen zur gemeinsamen weide treiben können. Wenn es in $\S 32$ heisst: 'Heribannum soluendum .I. ad rosbannum', so ist dies nach \$31 zu verbessern. Einen 'heerbann' haben die Friesen bekanntlich niemals gezahlt. Es kann hier nur von herba die rede sein. Hinter 'soluendum' stand in der vorlage des schreibers nicht .I., sondern .i., die regelmässige abbreviatur fur id est! Herbam soluendam id est ad rosbannum passt genau zu dem in $\$ 31$ uber den dritten zins bemerkten. Dieser zins hiess friesisch rosban; und jenes lateinische rosbannare beweist, dass es auch ein von rosban abgeleitetes schwaches verbum *rosbannia, * rosbanna (aus *rosbannjan) 'den rossban zahlen' im altfriesischen gegeben hat.

4. Ratforst. Der vierte zins hiess ratforst, plur. ratforstar. Die friesischen rechtsquellen kennen das wort ebenso wenig wie rosban. Ich weiss nicht, wie der erste teil desselben im altfriesischen gelautet haben mag. Sicher ist, was man unter ratforst zu verstehen hat. Es ist die abgabe, die fur die benutzung von wald, busch- und strauchwerk zu zahlen ist. Wie anderwärts, so wurde auch in Friesland der wald zur holzgewinnung und zur weide (eichelmast) benutzt.

BRESLAU.

HUGO JAEKEL.

\section{ZUR ALTFRIESISCHEN PSALMENGLOSSE.}

In der Zeitschrift fur deutsches altertum 32,417 fg. hat J. H. Gallée bruchstucke einer altfriesischen psalmenglosse, die er im Drenther provinzialarchiv zu Assen entdeckt hatte, veröffentlicht und besprochen. Sie erregen nach zwei seiten interesse. Einmal vermehren sie den altfriesischen wortschatz um einige worte und wortformen, sodann aber sind sie ein beweis, dass die altfriesische litteratur sich doch nicht ganz auf juristische arbeiten beschränkt hat. Dank dem lebhaften interesse des stamines fur recht und gericht wurde ja die juristische litteratur stark vervielfältigt und ist so auf uns gekommen, wăhrend die tubrige in den wirren des 16. jahrhunderts zu grunde gieng. Um nun jene fragmente fur die 